\title{
Understanding the Cognitive Work of Nursing in the Acute Care Environment
}

\author{
Patricia Potter, PhD, RN, FAAN \\ Laurie Wolf, MS, CPE \\ Stuart Boxerman, DSc, CHE \\ Deborab Grayson, MSN, RN, COHNS
}

\author{
Jennifer Sledge, MSW \\ Clay Dunagan, $M D$ \\ Bradley Evanoff, MD, MPH
}

Objective: To combine human factors engineering techniques with qualitative observation of nurses in practice to analyze the nature of nurses' cognitive work and how environmental factors create disruptions that pose risks for medical errors.

Background: Few researchers have examined the nature of nurses' cognitive work while in practice with patients. Researchers have described the broad range of thinking processes required in the acute care work setting, but have failed to examine how such processes are conducted and influenced by the complex care environment. A combined research methodology enables researchers to better understand how the nursing process becomes disrupted and the potential influence of this disruption on the safe and effective care of patients.

Methods: An ethnographic study, using mixedmethodological approaches, involved 7 staff registered nurses. The quantitative and qualitative data collection included field observation and summarative interviews.

Findings: A high number of cognitive shifts and interruptions, and a nurse's cumulative cognitive load, create the potential for disrupting a nurse's attention focus during care of patients. A majority of interruptions occurred as nurses performed interventions, particularly medication preparation.

Conclusion: New attention must be given to how care systems and work processes complement or interfere with nurses' cognitive work.

The delivery of nursing care in an acute care setting involves a complex series of physical, as well as cognitive, activities. A staff nurse performs care activities in response to patients' changing clinical conditions and anticipated ongoing needs, physician orders, the nurse's pattern for organizing care, and the routines of a nursing unit. In such an environment, minute-to-minute clinical decisions must be made to ensure that patient care is responsive and appropriate. Understanding the complex nature of the cognitive work of nursing offers a new perspective for the analysis of the environmental conditions that create risk for errors or omissions in care.

The 1999 Institute of Medicine (IOM) report To Err Is Human: Building a Safer Health System ${ }^{1}$ created public concern regarding errors in the American healthcare system. Scrutiny has been focused on the nursing profession because of the role nurses play in monitoring and managing patients' healthcare. A nurse's role includes immediate detection and intervention when breakdown in care occurs to reduce adverse events for patients. ${ }^{2}$ In clinical decisionmaking studies, few researchers have examined the nature of nurses' cognitive work while in practice with patients. Researchers have described the broad range of thinking processes required in the acute care work setting, but have failed to examine how such processes are conducted and influenced by the complex care environment. ${ }^{3}$

As part of a 3-year grant from the Agency for Healthcare Quality and Research, an innovative study combining human factors engineering (HFE)

Authors' affiliations: Research Scientist (Dr Potter), Research Coordinator (Ms Sledge), Professional Practice, BarnesJewish Hospital, St. Louis, Mo; Ergonomist (Ms Wolf), BJC Corporate Health, St. Louis, Mo; Associate Professor and Director (Dr Boxerman), Health Administration Program, Clinical Research Nurse Coordinator (Ms Grayson), Associate Professor of Medicine (Dr Dunagan), Associate Professor of Occupational and Environmental Medicine (Dr Evanoff), Washington University, St. Louis, Mo.

Corresponding author: Dr Potter, Barnes-Jewish Hospital, 1 Barnes-Jewish Hospital Plaza, Mailstop 9030 604, St. Louis, MO 63141 (pap1212@bjc.org). 
techniques and qualitative observation was conducted to analyze working conditions contributing to medical errors in the acute care setting. The application of HFE techniques and qualitative observation together provide a powerful tool for examining the clinical activities involved in the nursing process. The combined methodology enables researchers to better understand nurses' cognitive work in an environment where adapting to variation in patient needs and environmental factors is critical. ${ }^{4}$ It also provides an understanding of how disruptions in the nursing process influence safe and effective care of patients.

\section{Clinical Decision Making}

Clinical decision making is a critical thinking competency that separates professional nurses from technical nursing personnel..$^{5}$ It is a problem-solving activity that focuses on recognizing and defining patient problems and selecting appropriate treatment interventions. ${ }^{3,6}$ The registered nurse $(\mathrm{RN})$ in an acute care setting is the healthcare professional who takes initial action when a patient's clinical condition changes, decides if a patient is experiencing complications that warrant notification of a physician, or determines if a nursing therapy is effective.

The clinical decision-making process requires careful reasoning so that the options for the best patient outcomes are chosen on the basis of the patient's condition and care priorities. Nurses perform clinical decision making within the framework of the nursing process, which is aimed at identifying, diagnosing, and treating actual or potential human responses to health and illness. ${ }^{7}$

\section{Factors Influencing Decision Making}

Clinical decision making is influenced by the nurse's knowledge and attention focus, as well as factors within the workplace, including obstacles, multiple goals, missing data, and behaviors surrounding care situations. ${ }^{8}$ The acute care environment poses numerous barriers to a nurse's ability to attend to a patient's changing needs and presenting clinical condition. This becomes further complicated when the nurse cares for multiple patients. To prevent poor outcomes from occurring, nurses anticipate, react, accommodate, adapt, and cope to manage complexity within a changing environment. ${ }^{8}$

Few studies have examined how factors within the acute care environment interact or influence the cognitive work of nursing. Such a perspective would offer insight into the extent that disruption of cognitive work leads to errors or omissions in care.
Research by Ebright and colleagues ${ }^{8}$ was the first to explore the specific cognitive factors driving $\mathrm{RN}$ performance and decision making and the strategies used by RNs to manage their work. Their research used a human performance framework to identify the gaps and discontinuities that distract RNs from focusing on critical clinical reasoning about patients. The Ebright study combined field observation and interviews of nursing staff.

\section{Human Factors Engineering}

The science of HFE has been used widely in industry to improve the operation of complex systems and to reduce cognitive errors related to poor personmachine interface. ${ }^{9,10}$ Objectives of HFE are to maximize human and system efficiency, human wellbeing, and quality of life. ${ }^{11}$ HFE techniques were used when hospitals underwent reengineering and were beneficial in improving healthcare processes, such as laboratory specimen acquisition and medication dispensing.

However, Pepitone ${ }^{12}$ warns that traditional HFE or control-based engineering methods for improving performance are unsuccessful in analyzing dynamic knowledge work such as nursing practice. The author suggests that this lack of success can be explained by the fact that the work of nursing is selfpaced, discretionary, and nonlinear. HFE techniques provide valuable data about the psychomotor activities of nursing care, time measurements, and motion patterns. However, a task focus analysis is less likely to capture the type of information that can be gathered from a patient focus analysis. An alternative method is needed to study the nature of the cognitive work involved in clinical decision making and patient care management.

Clinical reasoning and decision making enable nurses to analyze information relevant to patient care. A nurse who moves between multiple patient rooms to attend to patients' changing clinical situations engages in a recursive cognitive process that uses inductive and deductive cognitive skills. ${ }^{13}$ Qualitative observation combined with HFE techniques is an appropriate methodology for capturing select cognitive activities within the nursing process. The methodology reveals a nurse's assessment, interaction with patients, problem and priority identification, collaboration with care givers, interventions, and evaluation of the effects of interventions. By observing nurses performing the nursing process, a researcher can better understand the manner in which the nursing process is conducted and the variables within the workplace that disrupt nurses' cognitive work. 


\section{Data and Design}

This study was conducted at a large tertiary medical center in the Midwest. Following approval by the human studies committee, RNs were invited to participate in a study involving observation of their clinical care of patients during a day shift of care. A convenience sample of 7 RNs with a wide range of acute care experience and clinical background was enrolled in an effort to obtain a diverse, but richly detailed, set of cases and to identify important patterns in RNs' care practices. The RNs' experiences as staff nurses ranged from 5 months to 31 years (average $=12$ years). The RNs worked on clinical areas including general medicine, general surgery, orthopedics, and neuromedicine.

A human factors engineer and an $\mathrm{RN}$ researcher teamed together to shadow each staff nurse during 4 to 9 hours of patient care activities. Initial observations involved 9-hour time frames. Analysis of initial findings suggested that data of the same quality could be collected during shorter time intervals. This would also enable researchers to increase sample size and refine observational techniques. Thus, observational periods were reduced to 4 hours after the first 3 observations.

The shadowing commenced with the early morning change-of-shift report. This allowed the $\mathrm{RN}$ researcher to gather data pertaining to each patient that the staff nurse was assigned, allowing later qualitative analysis of anticipated patient care activities and priorities. Both researchers then shadowed the staff nurses throughout the course of the observational period. The RN researcher intentionally queried each staff nurse only at the end of initial morning patient rounds so as to identify the staff nurse's perceptions of patient problems and care priorities. Otherwise, any interruptions resulting from the observers' presence were kept to a minimum. The RN researcher tracked and described all activities in the context of the nursing process, thereby capturing the cognitive intent of all care activities. The human factors engineer timed and categorized all activities on the basis of a task analysis framework.

The combined methodology for the study generated a rich volume of both quantitative and qualitative data. The 2 researchers met after each observational period to combine data and confirm findings. In addition, a group of expert clinicians, educators, and researchers met to interpret the qualitative data and to identify common themes describing the nurses' practice. Data for each RN included a qualitative summary of care activities, a task analysis, cognitive pathway, and computations of inter- ruptions, time spent with patient, omissions in care, and cognitive measures.

\section{Findings}

The 7 RNs were observed for a total of 43 hours. The RNs' care activities and percentage of time spent on each activity were categorized as follows:

- patient contact $(25 \%)$

- consultation $(26 \%)$

- documentation $(23 \%)$

- medication preparation and administration $(16 \%)$

- searching $(5 \%)$

- break time $(5 \%)$

Patient contact included assessment and all direct care activities, excluding medication administration. Searching involved RNs looking for resources such as supplies, equipment, or other staff members. The distribution of tasks was very similar to that analyzed from a previous task analysis conducted at the hospital.

Data from the HFE analysis was used to create a link analysis. A link is the sequence or connection between 2 elements of a task (eg, preparing a medication and delivering it to a patient). As an example, an analysis of the patterns of an RN's movement showed a total of 128 links with 2 heavy traffic patterns of 9 and 10 identical links (Figure 1). There were 10 identical links showing the $\mathrm{RN}$ going back and forth between patients $17 \mathrm{a}$ and $17 \mathrm{~b}$. The numbers of links suggest repetitive motion. Ordinarily, HFE analysts would look for ways to reduce repetitive steps through process or system changes (eg, redesign of supply access). However, qualitative data revealed that the frequent links between patients $17 \mathrm{a}$ and $17 \mathrm{~b}$ were attributed to frequent changes in both patients' clinical conditions.

The average number of links per hour made by the $\mathrm{RN}$ in Figure 1 was 13. That is, on the average, the RN walked from one location to another 13 different times every hour. The average number of activities per link was 1.9. Every time the nurse moved to a new location, she would perform approximately 2 activities before moving on to the next location. Similar findings were found for all 7 nurses, confirming nurses' practice of multitasking.

\section{Cognitive Pathway}

The most innovative aspect of this study, and one that garnered invaluable data for understanding the nature of nurses' work, was the cognitive pathway (Figure 2). The pathway is a unique visual graphic 


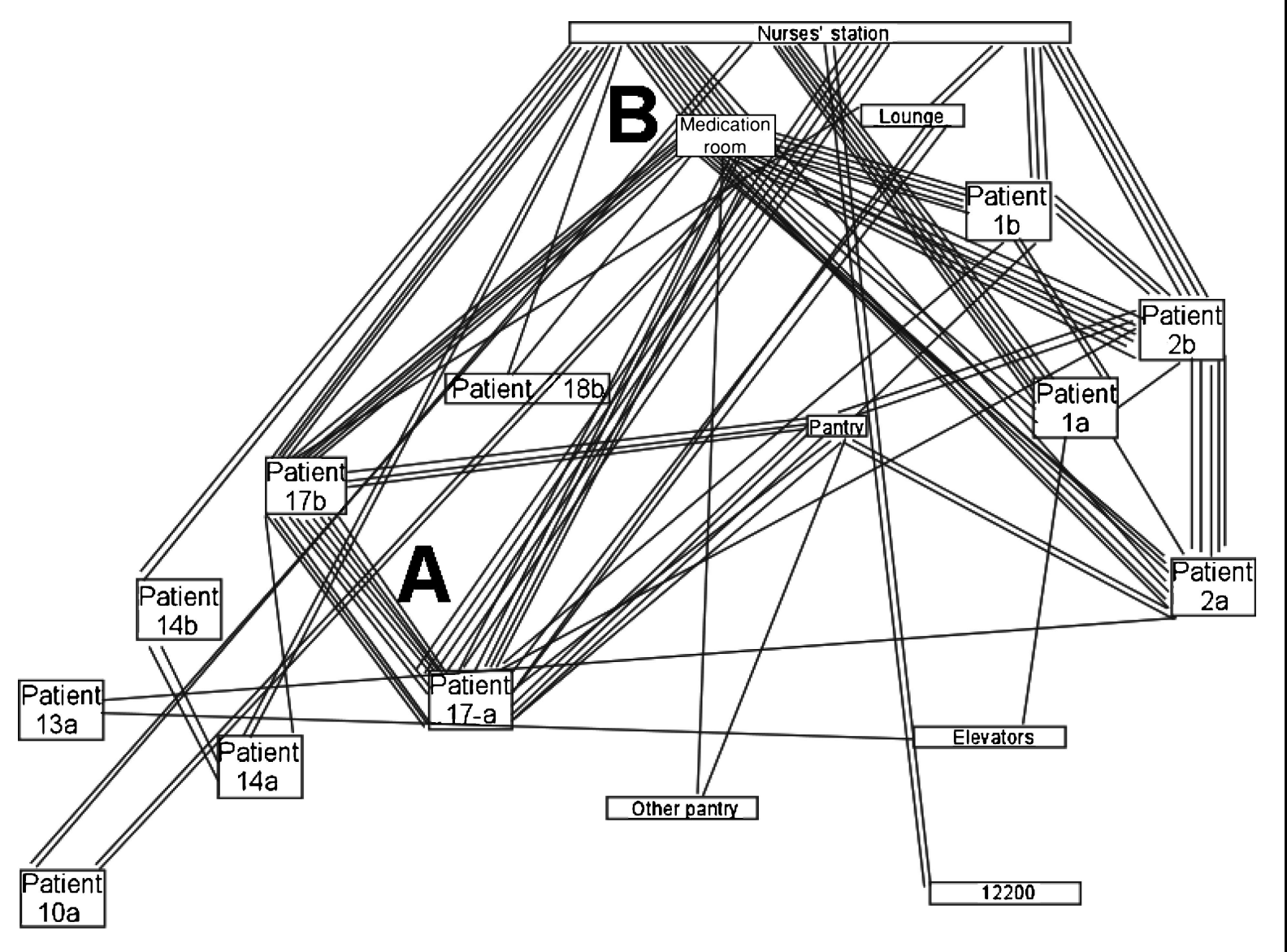

Figure 1. Example of a link analysis: Representing a registered nurse's movement between patient rooms and key geographic areas on a nursing unit.

that sequences steps of the nursing process as they are conducted for a given patient and across multiple patients over an observational period. The pathway also incorporates the occurrence of interruptions across time.

The vertical column on the left of the pathway designates the patients assigned to a given RN. The top horizontal column displays an observational time line. The pathway itself consists of a step-bystep accounting of each nurse's activities, labeled by steps of the nursing process: (1) assessment, (2) nursing diagnosis, (3) planning, (4) intervention, and (5) evaluation. A horizontal hash mark between each step of the process depicts the flow from one step to another. Note that there are no discontinuations of the process steps except for the occurrence of breaks or meal times. When process steps for multiple patients are bundled together, as is shown on the pathway at 11:25 AM, this represents the nurse conducting the same activity (eg, checking orders, completing patient classifications) for an entire set of patients at one time.
When a nurse shifted focus from one patient to another, this was designated a cognitive shift, depicted by the up and down arrows on the pathway. The nurse depicted in Figure 2 conducted 86 cognitive shifts over 9 hours of observation. A cognitive shift is a shift in attention from one patient to another during the conduct of the nursing process. Findings show that nurses are appropriately responsive to care demands, shifting attention as patient needs change, new procedures are ordered, or environmental processes interact. However, the number of shifts that occur in a given observation period are high, reflecting the frequent change in attention focus. The 7 RNs averaged 9 cognitive shifts per hour, meaning an $\mathrm{RN}$ was required to refocus from one patient to another about once every 6 to 7 minutes.

\section{Interruptions}

Both the HFE and nurse researcher tabulated interruptions. The HFE defined interruptions as an 
activity that stops the $\mathrm{RN}$ from performing an immediate task. This definition aligned with the HFE researcher's focus of each activity or task being unique and, once stopped, constitutes an interruption. The nurse researcher defined interruptions as actions on the part of other staff or occurrences from the environment that disrupted the RN's performance of a nursing process activity.

The researcher focused on the intent of each activity interrupted and whether it was part of the nursing process for a patient. For example, if a doctor stopped a nurse in a hallway to give an update on patient status, the HFE classified it as an interruption. However, within the nursing process context, if the physician communicated information needed in the care of a patient assigned to the nurse, the activity was classified as a planning activity, specifically consultation, and not an interruption.

As a result of the different perspectives, the total number of interruptions observed by the 2 researchers was disparate. The HFE observed a total of 261 interruptions (average $=5.9$ per hour). In contrast, the nurse researcher observed a total of 151 interruptions (average $=3.4$ per hour). Qualitative analysis revealed $47 \%$ of the interruptions occurred as the nurses were performing interventions.

Interruptions were further analyzed by location and outcome. A high percentage of interruptions $(22 \%)$ occurred in the medication room during medication preparation. The HFE observed an average of 1.3 interruptions per hour in the medication room, while the nurse researcher observed an average of 0.8 interruptions per hour. Typical sources of interruptions within the medication rooms were staff inquiries, missing drugs or drug administration supplies, and phone calls or pagers alarming. No attempt was made by the nurses to control sources of interruptions during the medication preparation process. The occurrence of interruptions seemed to be an accepted part of the nurses' work.

The researchers also calculated the number of interruptions that preceded or, presumably, created a cognitive shift. The intent was to examine if an association could be found between when interruptions occurred and the incidence of errors or omissions in care. An unplanned cognitive shift resulting from an interruption was seen as a potential factor that might distract the nurse's attention from an important care activity. The nurse researcher observed a total of 36 $(24 \%)$ interruptions occurring immediately prior to cognitive shifts.

Despite the number of interruptions, no errors were observed among the 7 nurses and no direct association could be made between a cognitive shift and observed omissions in care. There was one "near miss," involving a nurse who initially failed to prepare all of a patient's ordered $10 \mathrm{AM}$ medications, but quickly discovered the omission in time to prepare the correct doses. When the RN prepared medications for this patient, there was an interruption as the $\mathrm{RN}$ removed medications from the automatic dispensing unit.

By tracking nurses' assessment, planning, and intervention activities, the researchers were able to identify whether omissions in care occurred. Omissions were recorded when a planned activity identified by the RN, designated in medical orders, or communicated during change-of-shift report was not implemented. For example, if an RN assessed a patient experiencing discomfort and failed to provide a pain relief measure, an omission was recorded. A total of 21 omissions were observed for the 7 RNs. In the case of those RNs who were only observed for 4 hours, the researchers contacted the RNs by phone later in the afternoon to confirm whether selected activities had been completed.

The mixed methodology allowed the researchers to compute a measure described here as cognitive stacking. A nurse must continuously anticipate and attend to the numerous tasks and priorities that must be completed for his or her group of assigned patients. Ebright and colleagues ${ }^{8}$ described stacking as a nurse's organizational skill in moving on to other care activities to prevent down time when the nurse is not able to complete a care process. The researchers calculated a cognitive stacking measure, defined as an accumulative measure for quantifying tasks and priorities a nurse needs to perform at any given time for a group of patients during an assigned shift.

The cognitive stacking measure was calculated by tracking the patient care activities and priorities that accumulated over time as each nurse progressed through the period of observation. Any activity or priority identified as needing to be performed was added sequentially. When the RN completed an activity or priority, a number was subtracted from the measure. For example, at the beginning of a work shift, an RN might identify 3 care priorities for the first patient assessed and note that medications were to be given to 3 patients at $9 \mathrm{AM}$. The total priorities and patients requiring medications created a stacking measure of 6 . As the $\mathrm{RN}$ assessed a second patient and identified 2 priorities to be completed, the stacking increased to 8 . Once a patient received a 9 AM medication, the measure reduced to 7 .

The calculation of stacking provided a continuous measure that allowed researchers to identify the cognitive stacking load at any given time during the observation period. The time-weighted average for cognitive load for all RNs was 11 activities. The 
maximum number of activities stacked by $\mathrm{RNs}$ at any given time during the observations averaged 16 .

\section{Discussion}

The cognitive pathway revealed that the work of nursing is complex and nonlinear. Nurses engage in multiple cognitive shifts across patients throughout an assigned period of care. Cognitive shifts are both planned (eg, conducting morning rounds for each patient) and unplanned (eg, a nurse's change in focus from a stable patient to a patient whose condition worsened). The frequency of cognitive shifts creates a risk for the nurse to lose attention or cognitive focus for any one patient, depending on the occurrence of interruptions, the nature of any one cognitive shift (eg, patient deterioration), and the general pace of activities on the nursing unit.

Loss of attention to a patient's needs may potentially lead to delay in treatment, omissions in care, or even error. No errors were noted among the 7 nurses observed. However, a total of 21 omissions in care were observed. Each of the nurses observed reported that their work shifts were not unreasonably busy or chaotic. Thus, the researchers were unable to observe a staff nurse during a period when assigned patients were undergoing rapid or unpredictable clinical change. Further investigation is needed.

\section{Cognitive Intent of Nursing Care}

A cognitive shift does not represent physical movement on the part of the RN; instead, it represents a conscious shift in thinking from one patient to another. The findings show that a nurse remains cognitively focused on a given patient, regardless of whether the nurse is in the patient's room. This was clearly demonstrated when the researchers compared quantitative with qualitative measures for time spent by the RNs by location versus nursing process activity. The 7 RNs averaged 31 minutes spent in each patient's room over the observational time periods. In contrast, the RNs averaged 46 minutes being cognitively focused on each patient over the observational time periods.

These findings are significant in regard to verifying the dynamic nature of nurses' cognitive work while caring for patients. Using traditional HFE analysis alone fails to capture the cognitive intent of nurses' work and underestimates the time actually spent in important care activities. An underestimation of nurses' cognitive work misrepresents the nature of nursing practice. Administrative and management decisions regarding how nurses use their time should not be made solely on the basis of a task focus. The traditional underestimation of nurses' cognitive work may be a factor in failing to introduce management strategies that are successful in resolving the stress nurses experience with high patient acuity or low nurse-to-patient ratios.

\section{Patterns in Organizing Care}

Staff nurses follow unique patterns in the manner in which they organize and approach patient care. For example, some nurses began a shift of care by first conducting rounds on individual patients and then proceeding with medication administration and other treatment interventions. Other nurses first prepared all morning medications for assigned patients and then conducted patient rounds. One RN prepared all morning medications at one time, allowing her to engage in patient contact for a longer time frame during initial patient rounds and, thus, gather an extensive patient assessment.

The cognitive pathway also reveals the time nurses spend on each of the nursing process steps. For example, analysis showed that patient evaluation was the step of the nursing process performed most infrequently. Nurses consistently evaluated those patients who experienced clinical symptoms and changes. However, nurses did not perform ongoing evaluation of patients for activities such as medication monitoring or teaching outcomes. The cognitive pathway is a useful tool for evaluating nurses' methods for organizing care and conducting the nursing process. Data from pathways could prove useful for staff development and performance improvement initiatives.

\section{Impact of Interruptions}

Data from the task analysis showed that interruptions comprised, on average, $7 \%$ of the nurses' work time (based on nurse researcher's observations). The high incidence of observed interruptions poses important questions as to the extent such events disrupt a nurse's cognitive focus and ability to complete assigned care activities.

Nurses were frequently interrupted during interventional work (eg, administering medications, problem-solving intravenous infusions, and teaching patients). However, important assessment activities were also interrupted, potentially disrupting the nurses' ability to synthesize assessment findings and correctly identify patient problems. It was difficult for the researchers to connect a specific interruption or sequence of interruptions to an omission in care.

The nature of a nurse's work at the beginning of an assigned shift of care is to review each patient's 
priorities and to then proceed with an intended set of care activities. However, a patient's presenting clinical situation frequently changes and nurses must frequently reschedule care activities on the basis of waiting for processes or accessing resources. It thus becomes difficult to identify when certain activities should be completed and whether a specific interruption has disrupted those activities. Thus, it is difficult to match a specific interruption with a resultant omission in care.

A fourth of the interruptions that were observed by the $\mathrm{RN}$ researcher preceded a cognitive shift. This suggests that interruptions do move a nurse's attention from one patient to another, either because of the distraction created or because the nature of the interruption requires a redirection in care. Whether the occurrence of an interruption prior to a cognitive shift resulted in an omission could not be determined. Further study is needed to determine if there is an association between interruptions and omissions in care and whether there is a greater impact when interruptions disrupt a specific step of the nursing process.

\section{Medication Preparation Safety}

Safe medication preparation is a critical responsibility of staff nurses. Efforts at resolving medication errors often turn to the enforcement of drug administration policies and procedures. However, the context within which medication errors occur arguably prevents a nurse from applying the necessary concentration to adhere to the many steps of a policy. ${ }^{14}$

The number of interruptions that occurred within medication rooms disrupted RNs' attention during drug preparation and created a high risk for errors. The researchers observed that, in most cases, the medication rooms were highly visible locations with high levels of staff traffic. Nurses were interrupted while accessing dispensing systems, depositing medications in delivery containers, and confirming orders on computer screens. Study findings suggest that interventions are needed to eliminate or reduce interruptions that occur during the medication preparation process.

The ongoing cognitive load maintained by a nurse is critical to the nurse's ability to shift attention at any given time and recall the care activities requiring completion for a patient. In cognitive psychology, the concept of working memory capacity refers to the short-term cognitive system used for processing and storing information. ${ }^{15}$

An individual's working memory capacity is a gateway for a multitude of cognitive processes, including reasoning, knowledge, and strategy use. Working memory is much more than simply remembering a list of tasks. Researchers suggest that working memory is limited by a person's use of controlled processing, when information in memory must become or remain activated, particularly when distracting information competes for attention. ${ }^{16} \mathrm{~A}$ nurse must be able to think quickly, access previous knowledge as well as new information pertaining to a patient, and make judgments while performing care activities.

There has been no research to measure the working memory capacity of nurses in practice. A cognitive stacking measure was calculated in this study to direct attention to nurses' cognitive demands. The nurses observed in this study had seemingly high cognitive loads. The question is: to what extent cognitive load, interruptions, and frequent cognitive shifting interact to influence the ability to attend and respond to clinical situations.

Findings from this study cannot be generalized because of the small nonrandomized sample. The qualitative observation of one observer lends a degree of bias to the interpretation of nursing activities. However, the observation of nurses in practice offers a rich source of data from which a clearer understanding of how nurses engage in clinical decision making can be obtained.

\section{Conclusion}

Findings from this small study support the future use of mixed HFE and qualitative methodologies to better understand factors related to the clinical activities of nurses in practice. This study describes an aspect of nurses' work that until recently has not been examined in relation to the potential for causing errors in patient care. Although no errors were observed in this study, omissions in care were seen and numerous factors having the potential for disrupting nurses' cognitive work were observed.

The nature of a nurse's cognitive work is dynamic. A nurse conducts the nursing process in the face of changing patient needs, procedural demands, and environmental disruptions. The causes for errors or omissions in care remain ill defined in the literature. However, new attention must be given to how care systems and work processes complement or interfere with nurses' cognitive work. At critical moments, a nurse's cognitive load and ability to attend to a patient situation can be seriously compromised. There is a need to further explore the nature of nurses' cognitive work and how, during a time of critical activities, appropriate models of staffing or systems can be employed. 
1. Kohn LT, Corrigan JM, Donaldson MS. To Err Is Human: Building a Safer Health System. Committee on Quality of Healthcare in America. Washington, DC: National Academy Press; 2000.

2. Benner P, Sheets V, Uris P, Malloch K, Schwed K, Jamison D. Individual, practice, and system causes of errors in nursing, J Nurs Adm. 2002;10(32):509-523.

3. Smith Higuchi KA, Donald JG. Thinking processes used by nurses in clinical decision making. $J$ Nurs Educ. 2002;41(4):145-153.

4. Potter P, Boxerman S, Wolf L, et al. Mapping the nursing process: a new approach for understanding the work of nursing. J Nurs Adm. 2004;34(2):101-109.

5. Kataoka-Yahiro M, Saylor C. A critical thinking model for nursing judgment. I Nurs Educ. 1994;33(8):351356.

6. Gordon M. Nursing Diagnosis: Process and Application. 3rd ed. St. Louis: Mosby; 1994.

7. American Nurses Association. Nursing's Social Policy Statement. Washington, DC: The Association; 2003.

8. Ebright P, Patterson ES, Chalko BA, Render ML. Understand- ing the complexity of registered nurses work in acute care settings. J Nurs Adm. 2003;33(12):630-638.

9. Salvemini AV. Improving the human-computer interface: a human factors engineering approach. MD Comput. 1998;15(5):311-315.

10. Bologna LJ, Lind C, Riggs RC. Reducing major identification errors within a deployed phlebotomy process. Clin Leadersh Manage Rev. 2002;16(1):22-26.

11. Sanders MS, McCormick EJ. Human Factors in Engineering and Design. 5th ed. New York: McGraw-Hill Book Co; 1982.

12. Pepitone JS. A case for humaneering. IIE Solut. 2002;34(5): 39-44.

13. Simmons B, Lanuza D, Fonteyn M, et al. Clinical reasoning in experienced nurses. West J Nurs Res. 2003;25(6):701-719.

14. Armitage G, Knapman H. Adverse events in drug administration: a literature review. J Nurs Manag. 2003;11:130-140.

15. McNamara D, Scott JL. Working memory capacity and strategy use. Mem Cognit. 2001;29(1):10-17.

16. Tuholski SW, Engle RW, Baylis GC. Individual differences in working memory capacity and enumeration. Mem Cognit. 2001;29(3):484-492. 